\title{
Highly Multiplexed Optically Sectioned Spectroscopic Imaging in a Programmable Array Microscope
}

\author{
QUENTIN S. HANLEY and THOMAS M. JOVIN* \\ Department of Molecular Biology, Max Planck Institute for Biophysical Chemistry, Am Fassberg 11, D-37077 Göttingen, Germany
}

\begin{abstract}
We report the construction of a spectroscopic programmable array microscope capable of optical sectioning using Hadamard transform methods. Previously described Hadamard transform imaging consisted of first-order systems in which the mask was used for either illumination or detection. In this paper, a second-order Hadamard transform system is described in which a single one-dimensional mask serves for both illumination and detection. The system verifies earlier predictions that the optical spreading function along the transform axis can be inferred from the echoes arising in second-order systems using sequence lengths of $2^{\mathrm{n}}-1$. With a one-dimensional Hadamard mask, the system has an axial resolution of $1.3 \mu \mathrm{m}$ and may be used with or without the subtraction of an appropriately scaled wide field image depending on the degree of sectioning required. The system was tested using thin films, fluorescent beads, and a fixed biological specimen.
\end{abstract}

Index Headings: Hadamard transforms; Imaging spectroscopy; Point spread function; Line spread function; PSF; LSF; Echo correction; S-matrices; Spectral imaging; Second-order masking; Sampling; Hadamard transform spectroscopy.

\section{INTRODUCTION}

Optically sectioned spectroscopic measurements are of considerable interest for the examination of biological specimens. Such determinations are still performed by point or line scanning methods, for which significant improvements in speed and quality have been recently reported. ${ }^{1}$ What have been lacking, however, are spatially multiplexed techniques allowing simultaneous observation of large sample areas. Hadamard transform methods have the capability to supply this spatial multiplexing for optical sectioning systems, as described here.

Hadamard transform techniques are widely applicable and have been the subject of considerable recent development. They have been applied to capillary electrophoresis, ${ }^{2}$ mass spectroscopy, ${ }^{3}$ spectral imaging, ${ }^{4}$ electrode arrays, ${ }^{5}$ microscopy,, 7 and magnetic resonance imaging. ${ }^{8,9}$ Prior Hadamard methods in optical spectroscopy and for spectroscopic imaging in microscopes have been exclusively wide field techniques in which the Hadamard mask is used for encoding either on the illumination or the detection path, but not for both. An optical arrangement in which both the illumination path and the detec-

Received 4 April 2001; accepted 14 May 2001.

* Author to whom correspondence should be sent. tion path are encoded, a so-called second-order arrangement, has been proposed ${ }^{10}$ and the characteristics of such a system have been simulated. ${ }^{11}$

Concurrent with these developments has been a broad effort to improve the throughput and resolution of optical sectioning microscopes in both single and multi-photon methods. ${ }^{12-17}$ Among the many approaches, one of the more intriguing is the use of pseudo-random modulation of the apertures. ${ }^{18}$ Hadamard methods are of particular interest in this regard because the matrices used for Hadamard encoding are pseudo-random along any row or column. This characteristic has been used previously to generate optical sections. ${ }^{13,19}$

Combining Hadamard imaging spectroscopy with an optical sectioning method provides a unique opportunity to combine the capabilities of both approaches in a single instrument. This paper describes the construction and behavior of an optically sectioning Hadamard transform spectroscopic system. It is the first experimental implementation of a second-order Hadamard transform approach and verifies previously reported simulations of spreading in such a system. The spreading behavior was tested on defined specimens consisting of fluorescent beads and then applied to a biological sample. The optical sectioning behavior was verified using a thin fluorescent film.

\section{THEORY}

Pseudo-Random Sectioning Methods. A single point scanning confocal microscope allows the reduction of out-of-focus signal through the use of an illumination and a detection pinhole (Fig. 1a). Such a system may be parallelized by the use of a rotating disk and replacing the single pinhole of the standard confocal microscope with an array of pinholes (Fig. 1b). The first realization of a confocal microscope was based on the latter principle. ${ }^{20}$ However, its disadvantage is that the pinholes in the array must be placed sufficiently far apart to reduce cross-talk between pixels to a minimum, a condition that typically leads to $1 \%$ or less of the disk area to be comprised of apertures. ${ }^{21}$ The key feature of this cross-talk is that it contributes a correlated signal arising from a regular sequence of apertures used for scanning. Correlated crosstalk cannot be easily removed from an image. 


\section{a}

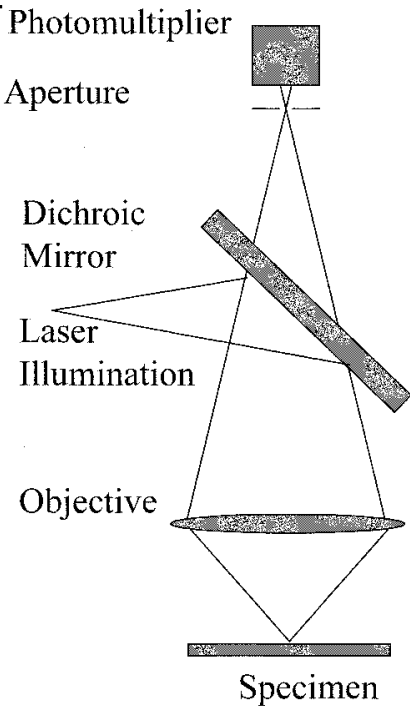

C

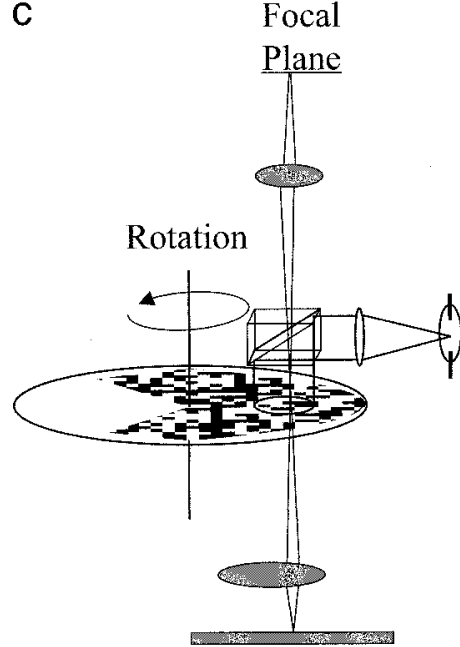

b

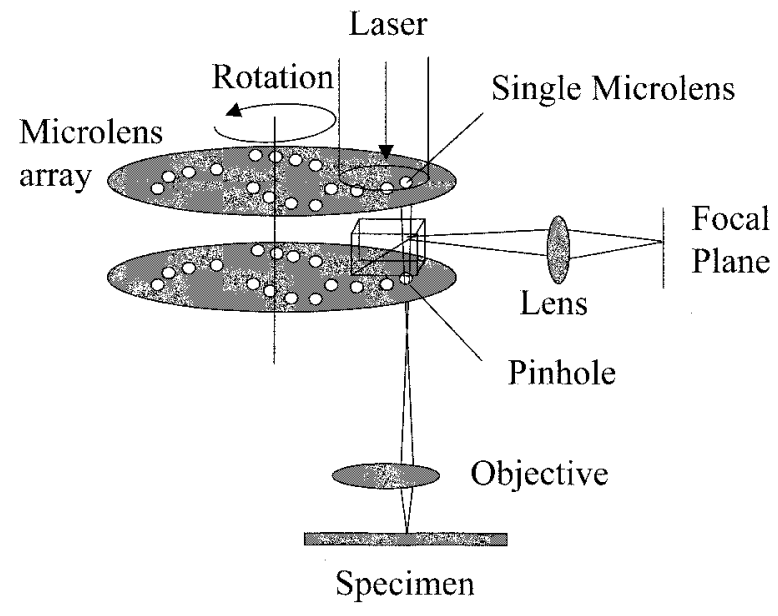

Lens

White light source

Pinholes

Objective

FIG. 1. Non-spectroscopic optical sectioning methods with a progressively greater degree of multiplexing. The most common optical sectioning microscope is the confocal scanning microscope (a). This instrument uses an illumination and detection aperture pair to reject out-of-focus blur. A rotating disk microscope (b) operates on a similar principle except that multiple apertures are used to generate an image. The sample is scanned by spinning the disk and observing the light returning through the apertures. This system forms a directly viewable image, but the multiple spots arranged in a regular array lead to a correlated aperture cross-talk signal. The degree of cross-talk has a complicated dependence on the wavelength, the numerical aperture, the pattern spacing, and the magnification. The system illustrated uses microlenses to make more efficient use of the illumination source. Similar strategies have also been applied to two photon microscopes, including a novel time-multiplexed approach. ${ }^{15-17}$ The correlated cross-talk can be removed and the aperture density increased to $50 \%$ by using a disk with randomly distributed apertures (c). The open sector of the disk is used to collect a wide field image that is scaled by $1 / 4$ and subtracted from an image taken through the random pattern. The difference is an optically sectioned image.

Random and pseudo-random sequences have been described previously ${ }^{12,22}$ as an approach for generating an optically sectioned image in a microscope. Here we only present the most important results of these earlier papers. If a sample is scanned with an array of apertures such that there is zero correlation between a given aperture and any other aperture, then the aperture cross-talk will be uncorrelated. The measured $\left(\mathrm{I}_{\mathrm{m}}\right)$ image consists of two components, a confocal component $\left(I_{c}\right)$ and an uncorrelated cross-talk component $\left(\mathrm{I}_{\mathrm{uc}}\right)$. $\mathrm{I}_{\mathrm{uc}}$, corresponding to a scaled wide field image $\left(I_{w}\right)$, may be removed by subtraction.

$$
\mathrm{I}_{\mathrm{c}}=\mathrm{I}_{\mathrm{m}}-f \mathrm{I}_{\mathrm{w}}
$$

where $f$ is a scaling factor. A rotating disk system scan- ning with random apertures can achieve optical sectioning with $\sim 50 \%$ of the surface area of the disk in the form of apertures (Fig. 1c). The zero correlation condition can be satisfied by modulating the apertures according to infinite random sequences, finite length Golay sequences, $\mathrm{S}$-sequences, and other orthonormal sequences. ${ }^{12,22}$ The $\mathrm{S}$-sequences are of particular interest here since they are the basis for Hadamard transform methods used for a wide range of imaging and spectroscopic applications. ${ }^{23}$ In addition, these sequences work well as pseudo-random sequences for optical sectioning in the programmable array microscope. ${ }^{13,19,24,25}$

Second-Order Hadamard Transform Methods. Standard Hadamard transform methods are a class of techniques in which a Hadamard matrix or an $\mathbf{S}$-matrix 

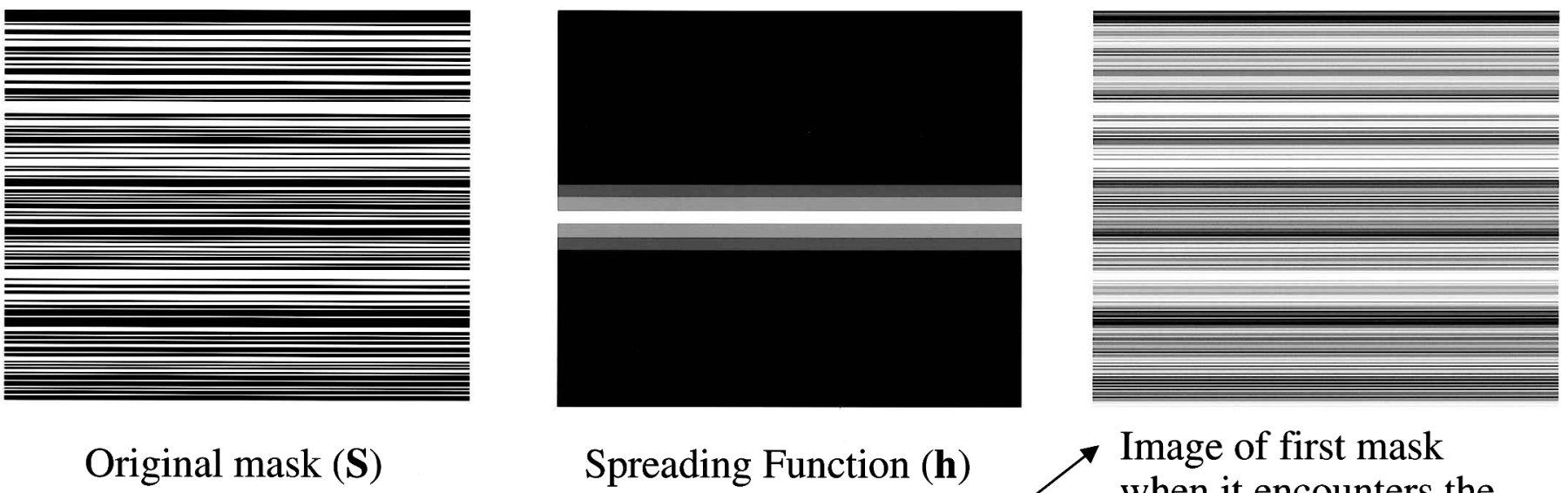

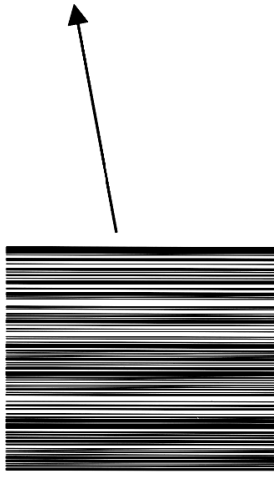

(a)

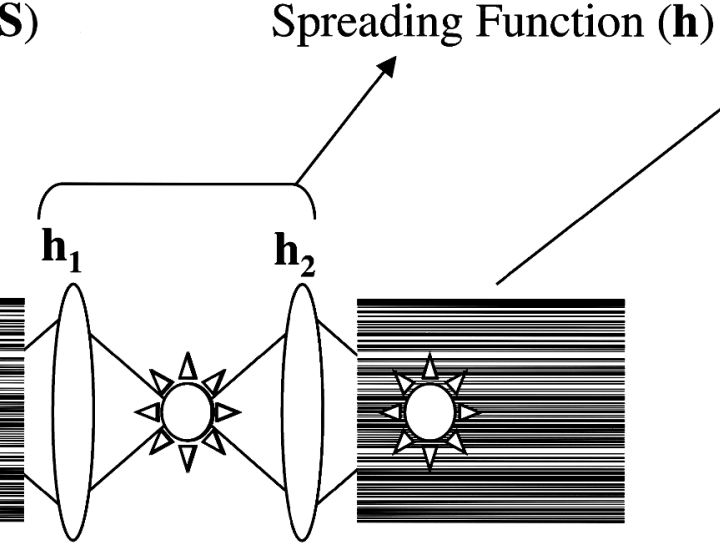

(b) (c) (e)
Image of first mask when it encounters the second mask (hS)

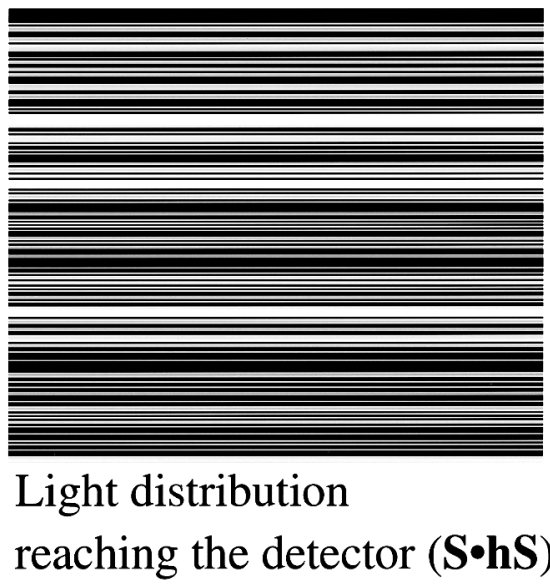

FIG. 2. Effect of an LSF on a one-dimensional second-order Hadamard transform instrument. A one dimensional mask (a) of length 255 is relayed by a lens (b) to an object (c). During this first relay step the mask image undergoes diffraction-limited spreading as defined by a lateral line spread function $\left(\mathbf{h}_{1}\right)$. In the case of a fluorescent object, the mask image stimulates fluorescence, which is collected by a lens (d) and relayed to a second mask (e). During this second relay process the image is once again modified by a lateral line spread function $\left(\mathbf{h}_{2}\right)$. The combination of $\mathbf{h}_{\mathbf{1}}$ and $\mathbf{h}_{\mathbf{2}}$ gives the overall spreading function (for clarity only the middle 30 points in the function are shown). The system is illustrated in a transmission arrangement having two separate masks. In the current implementation (see Fig. 3), the lens for excitation and detection was the same, as were the illumination and detection masks.

is used to generate a mask for encoding spatial, spectroscopic, or other information. The discussion here will be restricted to a cyclic subclass of $\mathbf{S}$-matrices, which have sequence lengths of $2^{\mathrm{n}}-1$. The Hadamard transform process can be divided into encoding (2) and transform (3) steps.

$$
\eta=\mathbf{S} \psi
$$

Then,

$$
\psi=\mathbf{S}^{-1} \eta
$$

A second-order Hadamard transform method is one in which the encoding step proceeds by the use of a single mask applied twice or the application of two separate masks. Second-order systems may be described mathematically as the point-wise matrix product $\mathbf{S} \cdot \mathbf{S}$. When no intervening process distorts $\mathbf{S}$, the point-wise product remains $\mathbf{S}$.

In practice, imperfect mask generation or the application of a point spread function (PSF) or line spread function (LSF) between the first and second masks leads to the generation of echoes in second-order Hadamard systems based on sequences of length $2^{\mathrm{n}}-1 .{ }^{11}$ In cases of spreading, the spreading function, $\mathbf{h}$, of the system is applied between the application of the two masks, resulting in encoding by a matrix $\mathbf{S}_{\mathrm{s}}=\mathbf{S} \cdot \mathbf{h S}$ that is no longer equal to $\mathbf{S}$. Echoes arise when the product $\mathbf{S}^{-1} \mathbf{S}_{\mathrm{s}}$ differs significantly from the identity matrix. Thus Eq. 2 becomes

$$
\eta_{\mathrm{s}}=\mathbf{S} \cdot \mathbf{h S} \psi
$$

$\psi$ may be recovered by applying the inverse matrix $(\mathbf{S} \cdot \mathbf{h S})^{-1}$, if it exists, to both sides of the equation.

$$
\psi=(\mathbf{S} \cdot \mathbf{h S})^{-1} \eta_{\mathrm{s}}
$$

This discussion assumes that the generation of the mask is perfect, a reasonable assumption for the case under study, and that all deviations from ideal behavior are due to the optical spreading function acting on the system between the application of the first and the second mask. A diagram of the second-order masking process is given in Fig. 2. If the mask is imperfect, the theory of distorted $\mathbf{S}$-matrices, which are designated $\mathbf{S}^{*}$, must be considered in order to recover $\psi$. $^{11,26-31}$

Transverse Line Spread Functions in Second-Order Hadamard Systems. Numerical calculations of wide field fluorescence point spread functions (PSF) have been 


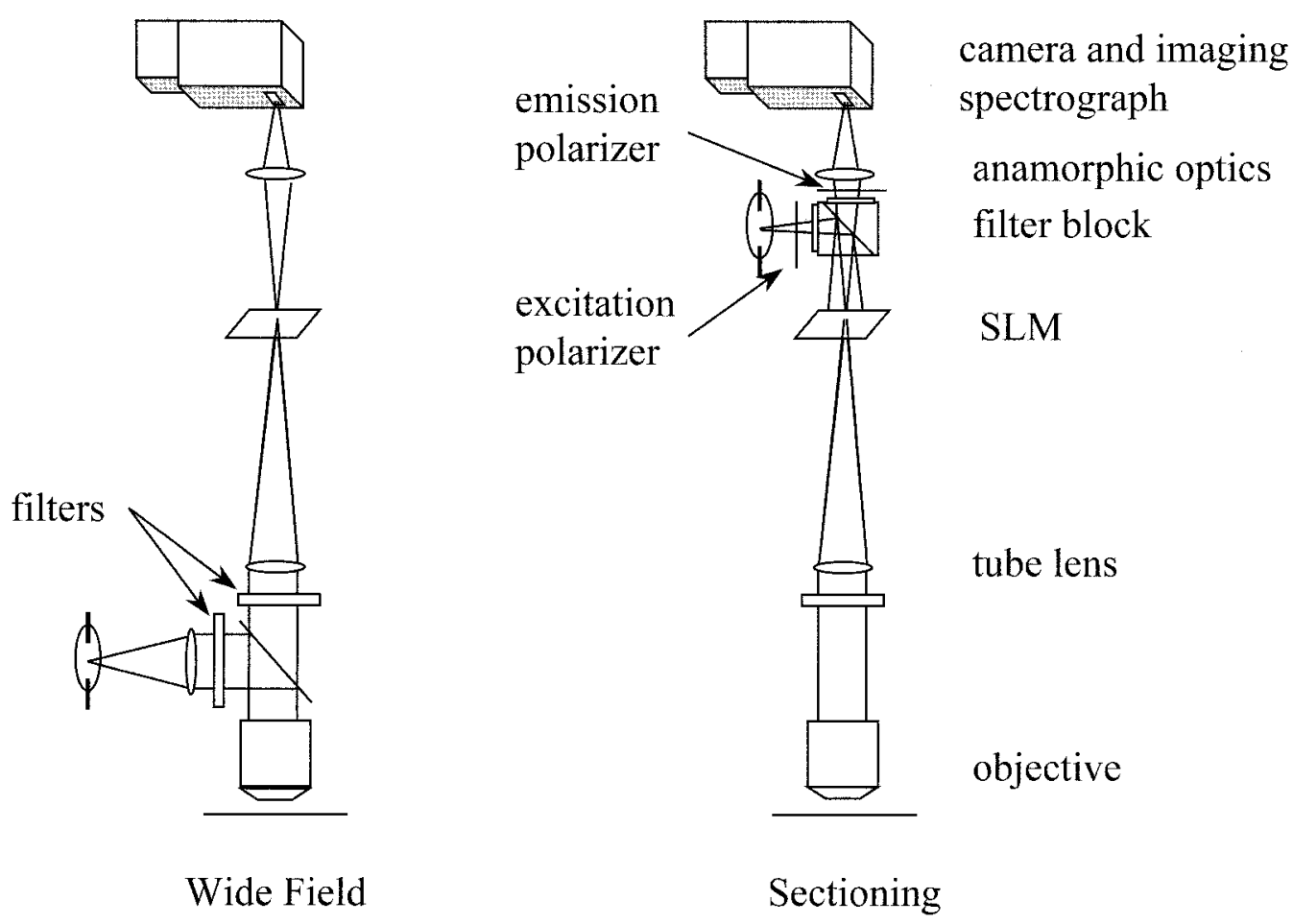

FIG. 3. Diagram showing a previously described wide-field ${ }^{7}$ (right) and the present (left) optical sectioning spectroscopic imaging system. The excitation and emission polarizers in the optical sectioning system are arranged in a parallel orientation to each other. Removing the excitation polarizer leads to a loss of optical sectioning allowing direct comparisons between sectioned and non-sectioned modes of operation. Upon changing from the wide field configuration to the optical sectioning arrangement, the upper polarizer from the SLM is removed and replaced with the excitation and emission polarizers. This modification reduces the fluorescent background and allows comparisons between wide field and sectioned images.

treated previously. We note, however, that analytical solutions to the fluorescence PSF are difficult, and commonly applied analytical solutions do not always give a satisfactory result. For these reasons we have adopted a previously described numerical approach. The computed wide field fluorescence PSF is a function of the spatial position $(x, y)$, refractive index, NA of the objective, wavelength of the light, and sampling. Details of these computations may be found in the literature. ${ }^{32,33}$

The line spread function represents the sum over an infinite series of PSFs displaced along the $x$ - or $y$-axes. In the case that the series is displaced along $y$, the line spread function can be obtained by integrating the PSF over $y$. This gives a wide field LSF.

$$
\operatorname{LSF}(x)=\int_{y=-\infty}^{y=\infty} \operatorname{PSF}(x, y) d y
$$

The treatment of $\mathbf{h}$ in Eqs. 4 and 5 is complicated by the combined effects of an excitation $\left(\mathbf{h}_{1}\right)$ and an emission $\left(\mathbf{h}_{2}\right)$ LSF (Fig. 2). The convolution of these two LSFs can be represented as the matrix product $\mathbf{h}_{1} \mathbf{h}_{2}$. The problem of interest is to recover the overall LSF by minimizing the echoes. In the case that $\psi$ is an impulse, such as might be observed with a bead specimen, the procedure consists of two iterative steps. First, the sum of squares, ss, for the $\psi_{i}$ not in the impulse is computed.

$$
s s=\sum_{i \neq \text { impulse }}^{\mathrm{n}}\left(\psi_{i}\right)^{2}
$$

Next a new overall LSF is generated and a new estimate of $\psi$ is computed.

$$
\psi_{\text {new }}=\left(\mathbf{S} \cdot \mathbf{h}_{\mathbf{1}} \mathbf{h}_{2} \mathbf{S}\right)^{-1} \eta_{\mathrm{s}}
$$

The new sum of squares is evaluated and the procedure repeated until a minimum is found. In the analysis of the echoes, we make the approximation that the two LSFs are equal and adjust the width in order to minimize ss. Other approaches to recovering the LSF could be envisioned, but are beyond the scope of the present study. Finally, it is important to note that $\mathbf{h}$ in Eqs. 4 and 5 is not a confocal LSF. Rather, $\mathbf{h}$ is a separate function representing the spreading of the first mask up to the point that it encounters the second mask. It is this spreading function that gives rise to echoes, not the confocal LSF.

\section{EXPERIMENTAL}

Optical System. The optically sectioning second-order Hadamard transform spectroscopic microscope system was based on a modified version of a previously described wide-field instrument (Fig. 3). ${ }^{7}$ The wide-field system was modified by moving the location of the illumination source such that the Hadamard mask served for both illumination and detection. The optical sectioning module was installed on a Nikon E-600 microscope equipped with a $100 \mathrm{~W} \mathrm{Hg}$ arc lamp and an epi-fluorescence unit. The spectroscopy module consisted of an SVGA format liquid crystal spatial light modulator (LCSLM) (Central Research Labs, England), anamorphic relay optics, a prism-based imaging spectrograph (PARISS, 
Lightform Inc., Belle Mead, NJ), and a CCD camera (KX-2, Apogee Instruments, Tucson, AZ). The LC-SLM consisted of a 3-component stack comprised of a lower polarizer, the liquid crystal matrix, and an upper polarizer. The liquid crystal matrix rotates the polarization of the incident light and the upper and lower polarizers are arranged parallel to one another. For the optical sectioning arrangement the upper polarizer was removed from the stack and placed above the illumination filter block following the emission filter. This reduced the fluorescence background from the polarizer. A second polarizer (Nikon) was installed in the illumination path between the arc lamp and the excitation filter of the filter block. Two points regarding this arrangement should be noted. First, the system only detects the parallel polarized component of fluorescence. Second, by removing the illumination path polarizer, an unsectioned image may be collected for direct comparison of sectioned and unsectioned arrangements. The atomic lines of a $\mathrm{HgNe}$ lamp provided wavelength calibration for the system. Further details may be found in the literature. ${ }^{4,7}$

Test Materials. A fluorescent thin film was prepared by application of a fluorescent highlighter marker to the underside of a microscope coverslip. By spreading the fluorescent marker material with a second coverslip, a highly robust bright fluorescent thin film was prepared. The thin film was observed with a $60 \times 1.4$ NA oil immersion objective. Fluorescently labeled latex beads with a diameter of $0.557 \mu \mathrm{m}$ (Fluorospheres Lot 6932-1, Molecular Probes, Eugene, OR) were dried onto the underside of a microscope coverslip and a drop of microscope oil was applied. The coverslip was then turned over and placed on a microscope slide and imaged with a $100 \times$ 1.3 NA oil immersion objective. A mounted $15-\mu \mathrm{m}$ acridine orange stained section of a Convallaria rhizome was used as a biological test specimen (Johannies Lieder GmbH, Ludwigsburg, Germany). All specimens were observed using a filter set consisting of a 470DF35 excitation filter, a 505DRLPO2 dichroic mirror, and a 515 EFLP emission filter (XF-19, Omega Optical, Brattleboro, VT).

Computation and Data Processing. Two approaches to the recovery of data encoded by $\mathbf{S}_{\mathrm{s}}$ were tested. The first method iteratively fit the individual coefficients of the spreading function $\mathbf{h}, \epsilon_{1}-\epsilon_{3}, \dagger$ on a data set obtained from a bead specimen. The sum of squares of the region of the image not containing the bead was minimized while adjusting the individual coefficients. Alternatively, h matrices were computed using algorithms described previously. The width of the LSF was adjusted while minimizing the sum of squares as defined in Eq. 7. Fitting an LSF is preferred since it requires only one parameter, the width of the LSF, to be fit, as opposed to the three required to fit the individual coefficients. Computation of the matrix inverse $(\mathbf{S} \cdot \mathbf{h S})^{-1}$ used the Gauss-Jordan elimination procedure with full pivoting using published algorithms. ${ }^{34}$

Axial Response Simulations. Simulations of the axial response consisted of full numerical computations as de-

\footnotetext{
$\dagger$ Here we follow the nomenclature of Hanley, ${ }^{11}$ in which $\mathbf{h}$ is a right circulant matrix generated from a vector consisting of an impulse and a set of coefficients, $\epsilon \pm i$, indexed relative to the impulse.
}

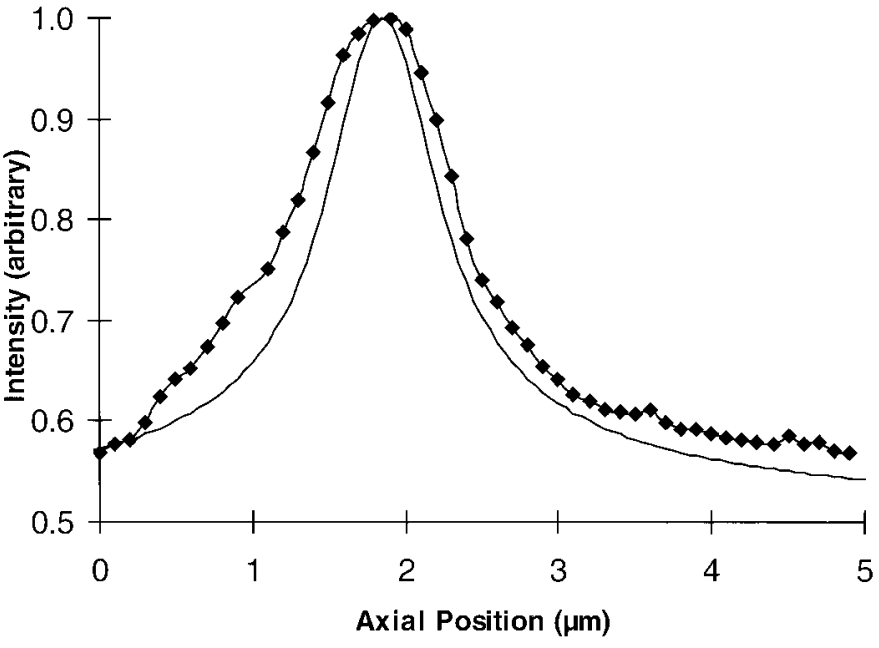

FIG. 4. Measured ( $\bullet$ ) and simulated (-) axial response of the optically sectioning system to a thin fluorescent film. The intensity represents the sum over all wavelengths plotted against the position of the thin film. The full width at half maximum was $\sim 1.3 \mu \mathrm{m}$, in good agreement with the predicted value of $1.1 \mu \mathrm{m}$. Note that even without subtraction of the uncorrelated offset, simply illuminating and detecting through the Hadamard mask was sufficient for restoring the missing cone to the optical transfer function (OTF).

scribed previously. ${ }^{13,22}$ The simulation assumed a line width of $33 \mu \mathrm{m}$ in the primary image plane, a $60 \times 1.4$ NA objective, excitation wavelength $480 \mathrm{~nm}$, and emission wavelength $540 \mathrm{~nm}$. The computation was performed using $8 \times$ oversampling of the $0.55 \mu \mathrm{m}$ projected line width.

\section{RESULTS}

Measured Axial Responses. The axial response to a fluorescent thin film gave a $z$-axis FWHM of $\sim 1.3 \mu \mathrm{m}$ (Fig. 4). This value was consistent with the value of 1.1 $\mu \mathrm{m}$ for the simulated response of a confocal line scanning system having a linewidth of $33 \mu \mathrm{m}$. This level of disagreement between simulated and measured axial responses is consistent with prior studies in high numerical aperture systems..$^{13,35}$ The uncorrelated offset was only slightly greater than $50 \%$, indicating excellent modulation of the axial response function. The uncorrelated offset is expected to have a limiting minimum value of $50 \%$ due to the characteristics of the S-sequence used to create the optical section. The shape of the axial response was slightly asymmetric, an effect most likely due to photobleaching of the test specimen. Note also that the simulation was for single excitation and emission wavelengths, whereas the measured data represent a sum over all wavelengths, which would be expected to result in a broadening of the response curve due to the lower resolution in the red.

The ability to produce an axial response to a fluorescent thin film in a highly multiplexed spectroscopic imaging system is of considerable importance. First, spatial multiplexing has the potential to increase the speed of acquisition relative to single-point or line-scanning procedures. Second, relative to wide-field instruments, only the areas that are under observation at any particular time are illuminated, resulting in lowered photobleaching of the sample in the plane of best focus. Finally, wide-field 


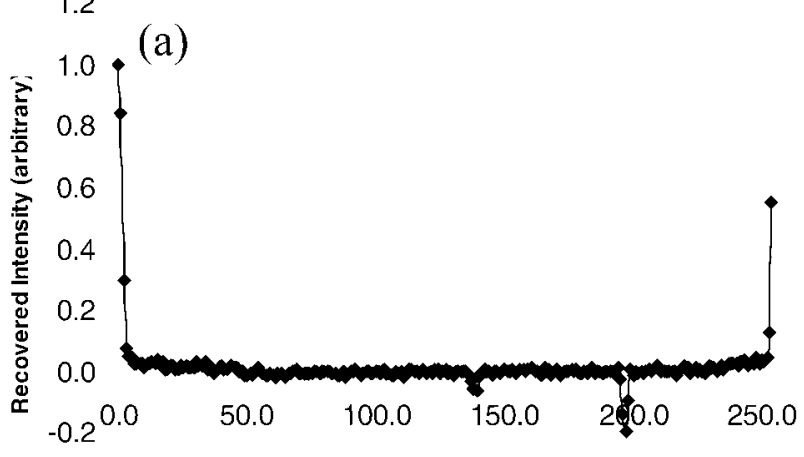

$-0.4$

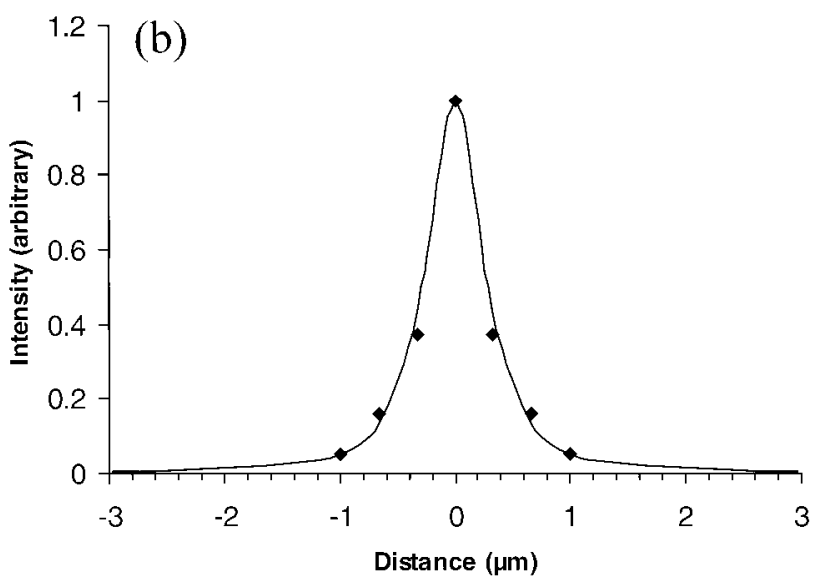

1.2

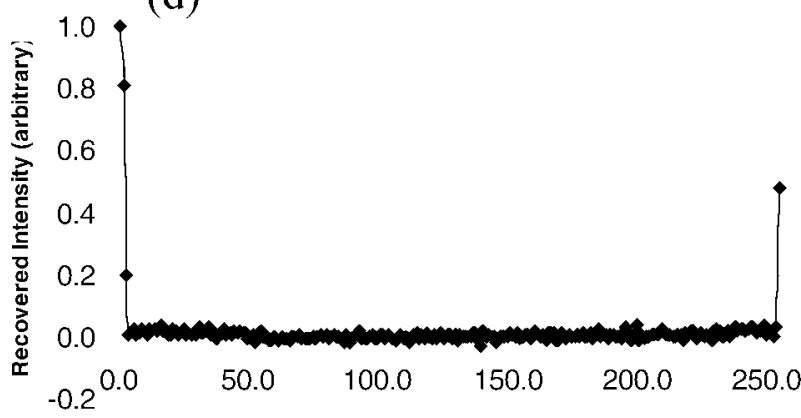

$-0.4$

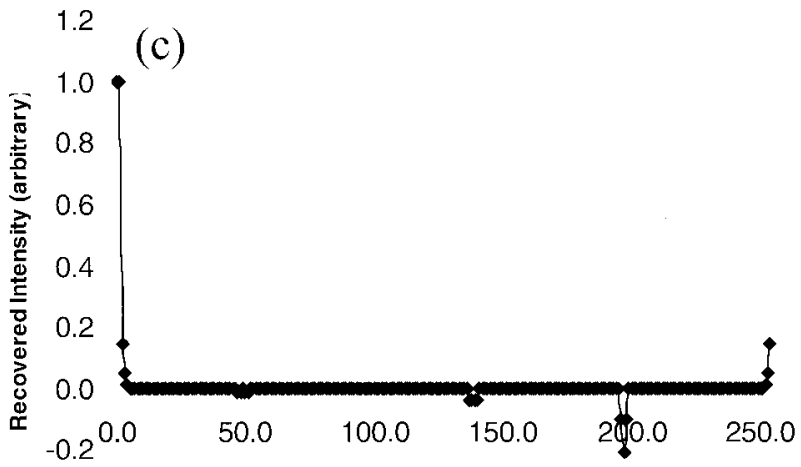

$-0.4$

Position on Transform Axis (arbitrary)

Position on Transform Axis (arbitrary)

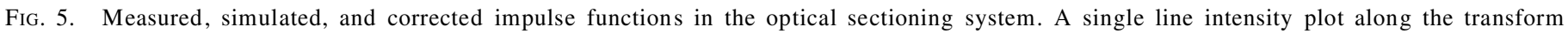

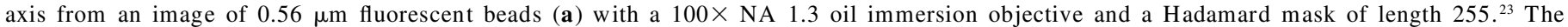

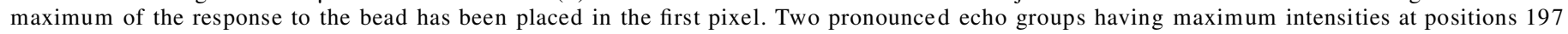

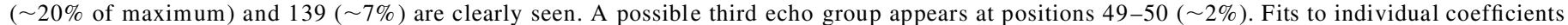

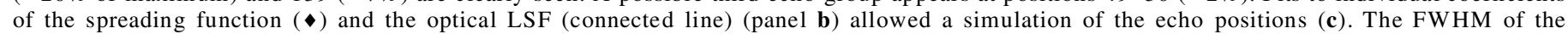

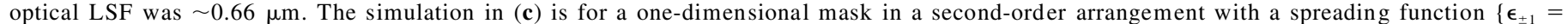

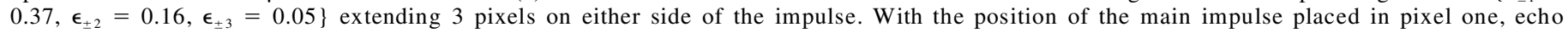

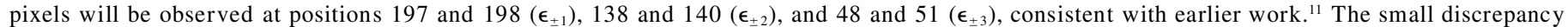

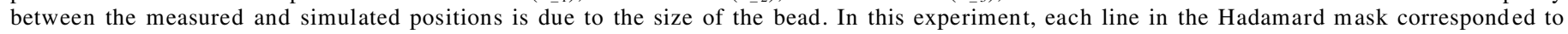
$0.33 \mu \mathrm{m}$. A corrected impulse from the bead after fitting the optical LSF to the data (d) removed the echoes.

methods suffer from a missing cone of information that makes it impossible to adequately position a plane in the axial direction. ${ }^{21}$ This missing cone of data also defines the degree to which image reconstruction methods can restore an image. However, as demonstrated in Fig. 4, simply illuminating and detecting through the Hadamard mask is sufficient to restore the missing cone of information, thereby better constraining subsequent image reconstruction. Finally, with the subtraction of a scaled wide-field image, an optical section is obtained directly, avoiding the acquisition of multiple focal planes, the storage of excessively large data sets, long acquisition times, and prolonged exposure leading to photo-destruction of the specimen.

Correction of Echoes: Simulation and Iterative Fitting of the Coefficients, $\boldsymbol{\epsilon}_{ \pm \mathrm{i}}$. A single line intensity plot along the transform axis from an image containing 0.56 $\mu \mathrm{m}$ beads exhibited 2-3 pronounced echo groups (Fig. 5a). The positions of maximum intensity of the echo groups were positions $197(\sim 20 \%), 139(\sim 7 \%)$, and $49-$ $50(\sim 2 \%)$, in agreement with the predicted positions
(197-198, $138 \& 140$, and $48 \& 51)$ previously reported for simulations of one-dimensional second-order Hadamard transform systems. ${ }^{11}$ Iterative minimization of the squared sum of intensities from positions 30-230 along the transform axis by symmetrically (e.g., $\boldsymbol{\epsilon}_{+3}=\boldsymbol{\epsilon}_{-3}$ ) adjusting the values of $\epsilon_{+1}, \epsilon_{+2}$, and $\epsilon_{+3}$ using the grid search approach yielded a spreading function $\{0.05,0.16,0.37$, $1,0.37,0.16,0.05\}$. Resimulation of the position and magnitude of the echoes using these coefficients gave a good representation of the observed echoes with discrepancies attributable to the finite size of the beads relative to an idealized single element impulse. Iterative fitting of the LSF resulted in a spreading function with a FWHM of $0.66 \mu \mathrm{m}$. Due to the lower number of parameters, this approach was used to estimate $\mathbf{h}$ and for correcting the echoes in full two-dimensional spectroscopic images. It should be noted that this recovered function represents the convolution of the illumination and detection LSFs along the transform axis.

The recovery of the spreading function using this procedure can be thought of as a calibration step for the 

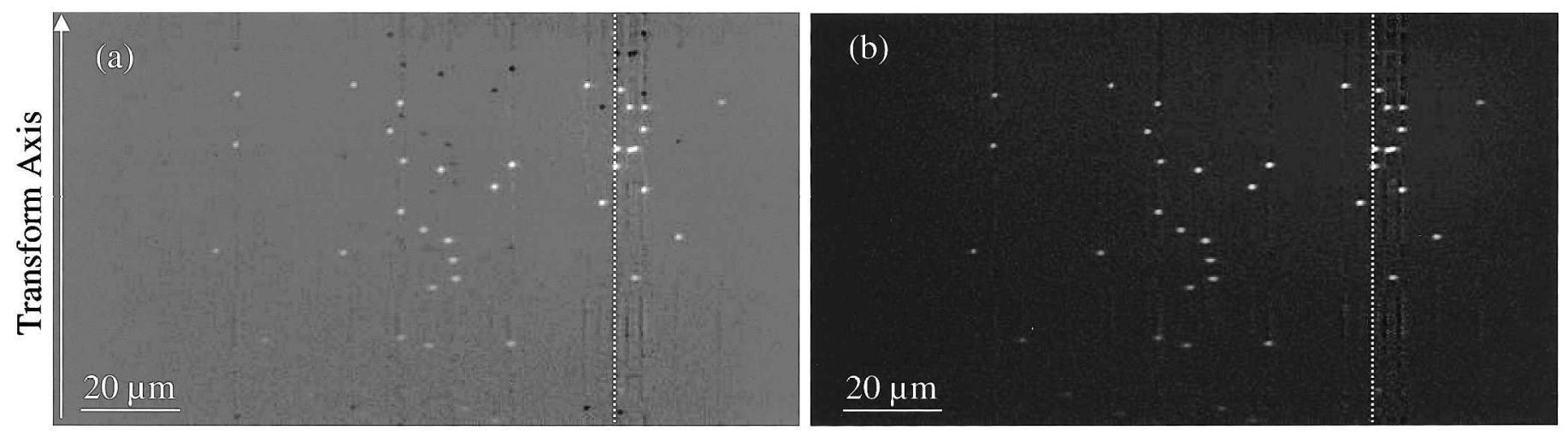

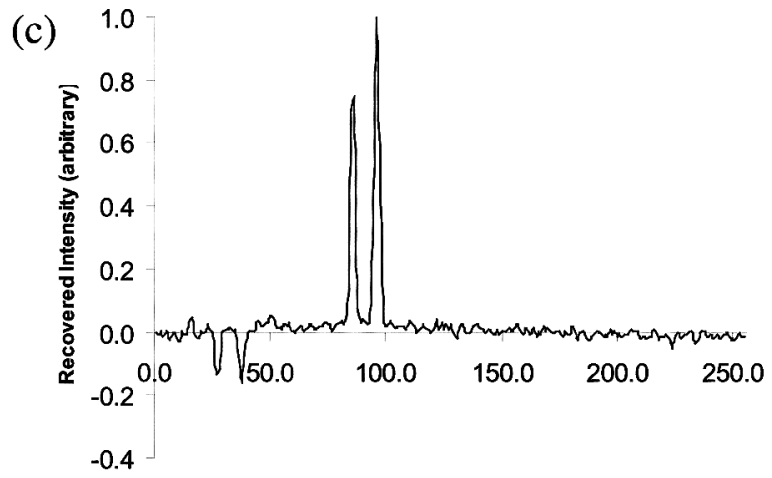

Position on Transform Axis (arbitrary) (d)

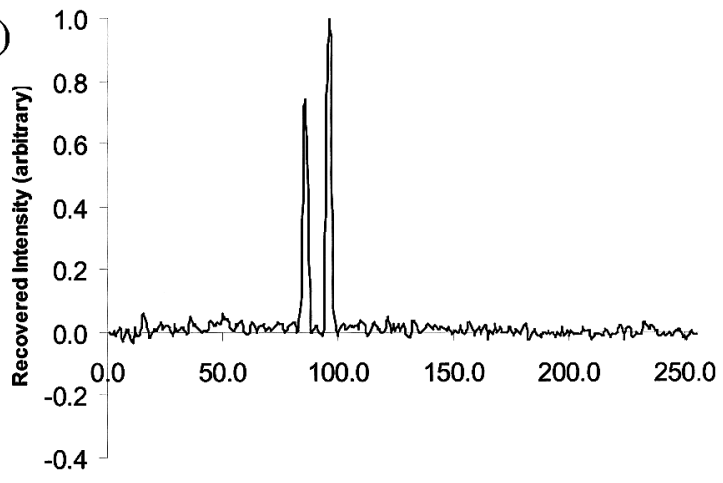

Position on Transform Axis (arbitrary)

FIG. 6. Image of a field consisting of $0.56 \mu \mathrm{m}$ beads taken with a $100 \times$ NA 1.3 oil immersion objective in the optically sectioning spectroscopic system before (a) and after (b) correction with the optical LSF shown in Fig. 5b. A single line intensity plot along the transform axis through two beads before correction (c) shows strong negative intensity corresponding to the echo groups simulated in Fig. 5c. After correction (d), a plot of the same line showed removal of the echo groups down to the noise floor of the measurement. Minor streaking along the transform axis was due to multiplexed noise from the high intensity of the beads being distributed along the transform axis and some minor photobleaching. These effects were not eliminated by the correction procedure.

second-order Hadamard system. Once the spreading function for the system is known, it can be applied to correct entire images.

Correction of Echoes: Two-Dimensional Spectroscopic Images. The correction obtained from the LSF was applied to the full two-dimensional spectroscopic image of the bead field (Fig. 6). Before correction, very strong negative echoes were present in the image. These echoes propagated only along the transform axis and were found exclusively in locations defined by the echo behavior of the particular sequence of length 255 used for the experiment. Application of the correction removed the echoes down to the noise floor of the measurement along the transform axis. This relatively simple procedure is sufficient for nearly every application of the system.

The bead samples represented a relatively difficult specimen for this type of analysis and are suitable for standardizing and correcting spectroscopic images taken near the bottom of the microscope cover glass. At this location the microscope objective is best corrected for aberrations. Fluorescence imaging with a high magnification objective provides the greatest challenge since the excitation power density and consequent photobleaching are maximized. It should be noted, however, that the magnitude of echo generation is related to the sampling density of the mask with respect to the projection of the LSF in the primary image plane. In a second-order ar- rangement, the echo magnitude will increase with the degree of over-sampling of the specimen by the mask. As noted previously, the $33 \mu \mathrm{m}$ pixels of the SLM are not sufficient to allow even Nyquist sampling of the Airy disk from the $100 \times 1.3 \mathrm{NA}$ objective. Although diffraction limited imaging effects are apparent in this data set, they are small compared to what would be expected from an image obtained by heavily over-sampling with the Hadamard mask. Finally, the ability to measure the spreading function of a microscope while observing a complex object is of interest. The extent to which the spreading functions obtained by the fitting procedure can assist other types of image analysis is presently unclear but will be the topic of continuing studies.

Imaging of Biological Specimens. The Convallaria rhizome was observed in wide-field (illumination polarizer out), raw-sectioned (illumination and detection through the Hadamard mask), and fully-sectioned (rawsectioned minus scaled wide-field) modes. The wide-field image exhibited the most blur (Fig. 7a), an effect most apparent in the spectral images. The raw-sectioned image contained less blur (Fig. 7b) and, prior to correction, exhibited echoes (not shown). The echoes were fully corrected after fitting the LSF. The fully-sectioned image contained the least amount of blur (Fig. 7c), resulting in reduction of the background by as much as a factor of 3 


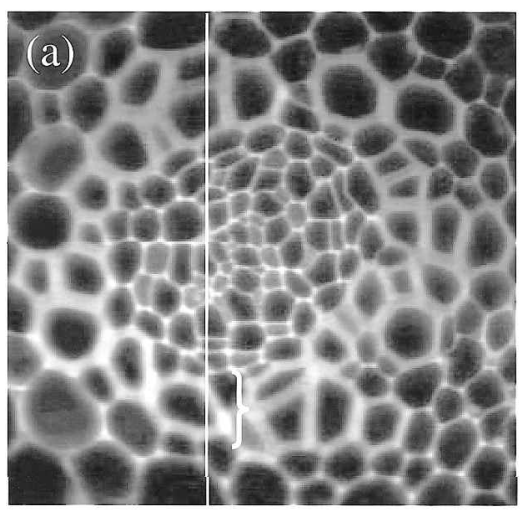

Wide Field

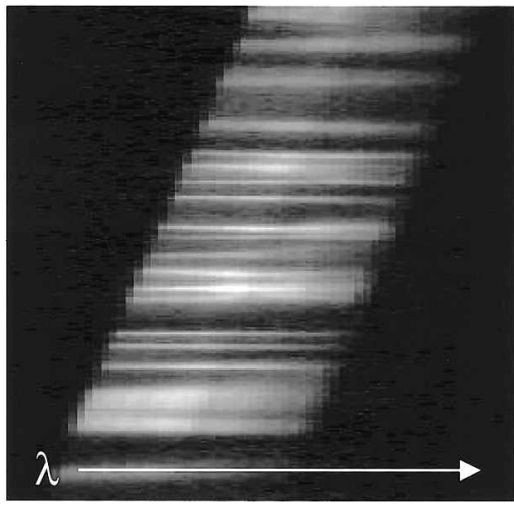

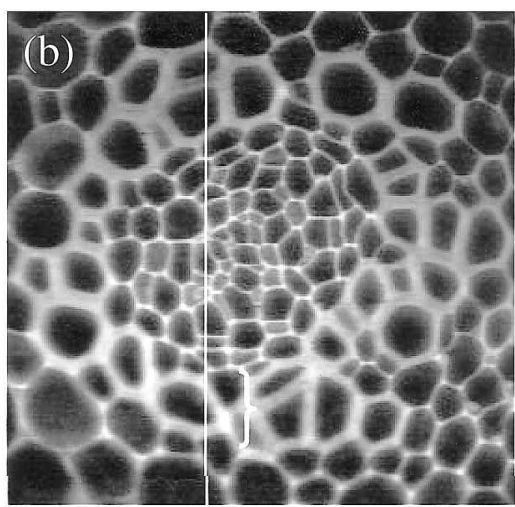

Raw Sectioned Image

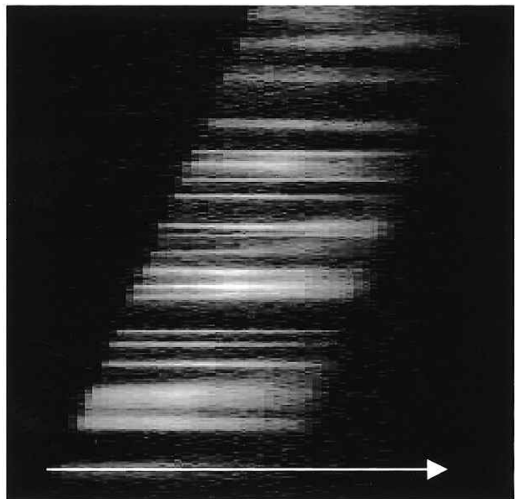

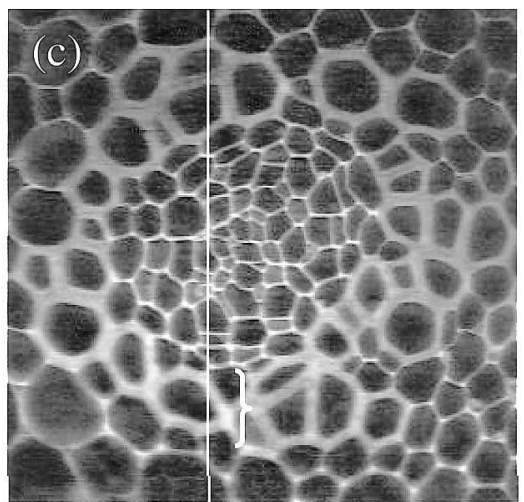

\section{Fully Sectioned Image}

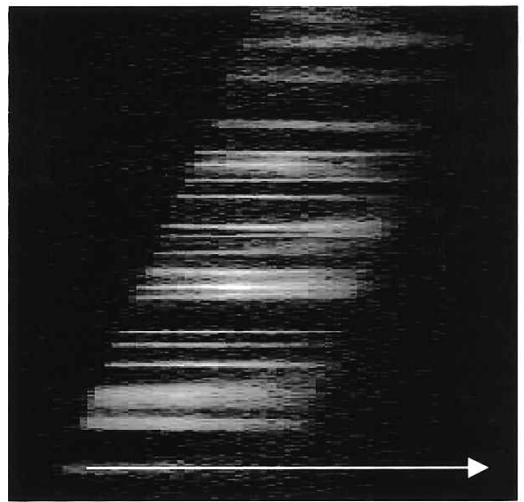

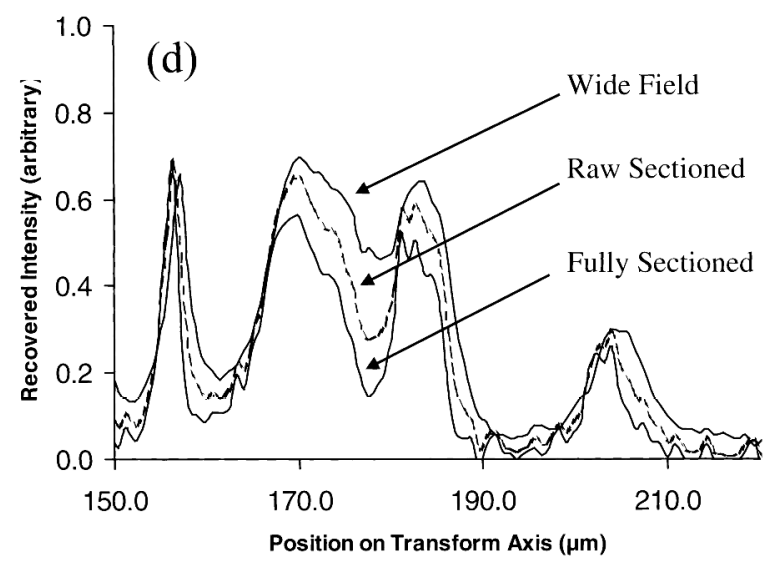

FIG. 7. Comparison of wide-field (a), raw-sectioned (b), and fully-sectioned (c) imaging modes. The wide-field image shows the largest amount of out-of-focus blur. This mode of imaging is incapable of assigning the axial position of a thin film. The raw-sectioned image shows a reduction in the amount of blur in the image. The fully-sectioned image obtained by subtracting an appropriately scaled wide-field image from (b) shows further suppression of the out-of-focus light most noticeable in the spectral images in the lower half of the figure. Both a reduction in background and an enhancement of the resolution are seen in this image. The line corresponding to the location of the spectral images is indicated with a line in each figure. For illustration, no shift correction was applied, giving a diagonal appearance to the spectral images. ${ }^{7}$ For computational simplicity, all the echo corrections were applied prior to the shift correction. To illustrate the improvement in background rejection, a section of the line (indicated with the bracket in the images) has been enlarged and plotted graphically (d).

(Fig. 7d). In all cases, the spectra obtained were consistent with prior measurements on this test sample.

\section{CONCLUSION}

Three reasons for using Hadamard methods are of interest in the context of this study. (1) They increase the throughput of an optical system, the primary consideration of the present approach. Hadamard systems provide inherent optical sectioning when used for both illumina- tion and detection. At the same time they make efficient use of the available light, and technical advances in programmable mask technology should yield further improvements. (2) Hadamard methods take advantage of the optimal experimental design represented by $\mathrm{H}$ and $\mathrm{S}$ matrices. ${ }^{23} \mathrm{~S}$-matrix designs are A-optimal for large $n$ and D-optimal. "A-optimal" indicates that the average mean squared error approaches the minimum possible for large $n$ and "D-optimal" that the volume of the region in 
which the estimates may be expected to lie is minimal. It is possible that better design matrices can be constructed for systems employing second-order masks in diffraction limited imaging. Adaptive optical mask technologies, such as that used here, might allow these more optimal designs, if they exist, to be realized for second-order systems with significant diffraction. It is clear, however, that as $\mathbf{h}$ approaches the identity matrix, I (e.g., systems with insignificant optical spreading), second-order systems are as optimal as first-order systems. (3) Hadamard transforms are computationally efficient. Under normal conditions the fast Walsh-Hadamard transform can be computed faster than the fast discrete Fourier transform. The matrix multiplication steps in Eqs. 5 and 8, while straightforward, do not make use of the fast Walsh-Hadamard transform. However, it is likely that more efficient procedures will be found, as has been the case for other corrections to Hadamard transform methods.

Illuminating and detecting through a Hadamard mask restores some of the missing cone of information lost in wide-field microscope arrangements, thus allowing the axial position of a thin film to be assigned without further processing. By adding the extra step of subtracting a portion of a wide-field image, a true optical section can be obtained. Under conditions in which the sampling density is high with respect to the Airy disk, information about the spreading function of the microscope between the application of the first and second mask may be obtained.

The use of the second-order Hadamard arrangements should allow efficient acquisition of sectioned images in a wide range of spectroscopic modes. These might include: (1) Raman and other luminescence techniques; (2) IR and near IR absorption; and (3) a variety of reflectance techniques in the IR, NIR, and UV-Vis. Hybrid techniques incorporating this approach, such as combinations of emission and lifetime spectroscopy, can also be envisioned. Finally, second-order Hadamard methods could be of interest in non-spectroscopic applications.

\section{ACKNOWLEDGMENT}

The authors would like to thank Dr. Peter Verveer of EMBL Heidelberg for helpful discussions of LSFs and for supplying algorithms for generating the wide-field fluorescent PSF.

1. N. M. Haralampus-Grynaviski, M. J. Stimson, and J. D. Simon, Appl. Spectrosc. 54, 1727 (2000).

2. T. Kaneta, Y. Yamaguchi, and T. Imasaka, Anal. Chem. 71, 5444 (1999).
3. A. Brock, N. Rodriguez, and R. N. Zare, Rev. Sci. Instrum. 71, 1306 (2000).

4. Q. S. Hanley, P. J. Verveer, D. J. Arndt-Jovin, and T. M. Jovin, J. Microsc. 197, 5 (2000)

5. R. S. Freire, J. J. R. Rohwedder, and C. Pasquini, Analyst (Cambridge, U.K.) 124, 1657 (1999).

6. H. W. Tang, G. Q. Chen, and Y. N. Zeng, Microchem. J. 61, 177 (1999).

7. Q. S. Hanley, P. J. Verveer, and T. M. Jovin, Appl. Spectrosc. 53, 1 (1999).

8. G. Goelman, Magnetic Resonance Imaging 18, 939 (2000).

9. O. Gonen, J. Murdoch, R. Stoyanova, and G. Goelman, Magnet. Reson. Med. 39, 34 (1998).

10. T. M. Jovin, Q. Hanley, and P. Verveer, Confocal Spectroscopy System and Method, US Patent 6128077, October 3, 2000.

11. Q. S. Hanley, Appl. Spectrosc. 55, 318 (2001).

12. R. Juskaitis, T. Wilson, M. A. A. Neil, and M. Kosubeck, Nature (London) 383, 804 (1996).

13. Q. S. Hanley, P. J. Verveer, M. J. Gemkow, D. Arndt-Jovin, and T. M. Jovin, J. Microsc. 196, 317 (1999).

14. M. A. A. Neil, R. Juskaitis, and T. Wilson, Opt. Commun. 153, 1 (1998).

15. J. Bewersdorf, R. Pick, and S. Hell, Opt. Lett. 23, 655 (1998).

16. T. A. Klar, S. Jakobs, M. Dyba, A. Egner, and S. W. Hell, Proc. Natl. Acad. Sci. U.S.A. 97, 8206 (2000).

17. A. Egner and S. W. Hell, J. Opt. Soc. Am. A. 17, 1192 (2000).

18. T. Wilson, R. Juskaitis, M. A. A. Neil, and M. Kozubek, Opt. Lett. 21, 1879 (1996).

19. Q. S. Hanley, P. J. Verveer, and T. M. Jovin, Appl. Spectrosc. 52, 783 (1998).

20. M. Petran, M. Hadravsky, M. D. Egger, and R. Galambos, J. Opt. Soc. Am. 58, 661 (1968).

21. J. B. Pawley, Handbook of Biological Confocal Microscopy (Plenum Press, New York, 1995).

22. P. J. Verveer, Q. S. Hanley, P. W. Verbeek, L. J. van Vliet, and T. M. Jovin, J. Microsc. 3, 192 (1998).

23. M. Harwit and N. J. A. Sloane, Hadamard Transform Optics (Academic Press, New York, 1979).

24, P. J. Verveer, Q. S. Hanley, P. W. Verbeek, L. J. van Vliet, and T. M. Jovin, J. Microsc. 189, 192 (1998).

25. R. Heintzmann, Q. S. Hanley, D. J. Arndt-Jovin, and T. M. Jovin, J. Microsc., paper in press.

26. R. A. Dyer, S. A. Dyer, B. K. Harms, T. W. Johnson, and J. B. Park, IEEE T. Instrum. Meas. 39, 163 (1990).

27. S. A. Dyer and J. B. Park, Appl. Spectrosc. 43, 278 (1989).

28. S. A. Dyer, B. K. Harms, J. B. Park, T. W. Johnson, and R. A. Dyer, Appl. Spectrosc. 43, 435 (1989).

29. T. W. Johnson, J. B. Park, S. A. Dyer, B. K. Harms, and R. A. Dyer, Appl. Spectrosc. 43, 746 (1989).

30. J. D. Tate, J. V. Paukstelis, R. M. Hammaker, and W. G. Fateley, J. Mol. Struct. 300, 385 (1993).

31. M. H. Tai, M. Harwit, and N. J. A. Sloane, Appl. Opt. 14, 2678 (1975).

32. P. J. Verveer, Ph.D. Dissertation, Delft University of Technology (1998).

33. H. T. M. van der Voort and G. J. Brakenhoff, J. Microsc. 158, 43 (1990).

34. P. M. Embree and B. Kimble, C Language Algorithms for Digital Signal Processing (Prentice Hall, Englewood Cliffs, 1991)

35. H. Jacobsen and S. W. Hell, Bioimaging 3, 39 (1995). 